\title{
HOST Study - HIV in Orthopaedic Skeletal Trauma Study: protocol for a multicentre case-cohort study
}

\author{
Graham SM${ }^{1}$, Harrison $\mathrm{WJ}^{2}$, Lalloo $\mathrm{DG}^{3}$, Simpson $\mathrm{AH}^{4}$, Laubscher $\mathrm{M}^{5}$, Held $\mathrm{M}^{5}$, Ferreira $\mathrm{N}^{6}$, Maqungo $\mathrm{S}^{7}$ \\ 1 Orthopaedic Trauma and Wellcome Trust Clinical Research Fellow; Department of Clinical Sciences, Liverpool School of Tropical Medicine, Liverpool, United \\ Kingdom; Department of Orthopaedic Surgery, University of Edinburgh, Chancellor's Building, 49 Little France Crescent, Old Dalkeith Road, Edinburgh EH16 4SB, UK; \\ Orthopaedic Research Unit, Department of Orthopaedic Surgery, Groote Schuur Hospital, University of Cape Town, Cape Town, South Africa \\ 2 Consultant orthopaedic surgeon; Countess of Chester Hospital, Chester, CH2 1UL, UK \\ 3 Professor and Dean of Clinical Sciences and International Public Health; Chair in Tropical Medicine; Director Wellcome Trust Liverpool Glasgow Centre for Global \\ Health Research; Department of Clinical Sciences, Liverpool School of Tropical Medicine, Liverpool, United Kingdom; Malawi-Liverpool-Wellcome Trust Clinical \\ Research Programme, Blantyre, Malawi \\ 4 Professor of Orthopaedics and Trauma, and consultant orthopaedic surgeon; Department of Orthopaedic Surgery, University of Edinburgh, Chancellor's Building, \\ 49 Little France Crescent, Old Dalkeith Road, Edinburgh EH16 4SB, UK \\ ${ }^{5}$ Consultant orthopaedic surgeon; Orthopaedic Research Unit, Department of Orthopaedic Surgery, Groote Schuur Hospital, University of Cape Town, Cape Town, \\ South Africa \\ ${ }^{6}$ Associate Professor and Head Clinical Unit: Tumour, Sepsis and Reconstruction; Division of Orthopaedics, Department of Surgical Sciences, Faculty of Medicine and \\ Health Sciences, Stellenbosch University \\ Professor and Head: Orthopaedic Trauma Service; Orthopaedic Research Unit, Department of Orthopaedic Surgery, Groote Schuur Hospital, University of Cape Town, \\ Cape Town, South Africa
}

Corresponding author: Dr SM Graham, Division of Orthopaedic Surgery, H49 OMB, Groote Schuur Hospital, Observatory, 7925, Cape Town; South Africa tel (+27 21) 406 6157/8; email: simonmatthewgraham@doctors.org.uk

\begin{abstract}
Background: Human immunodeficiency virus (HIV) and antiretroviral therapy (ART) have both been shown to reduce bone mineral density, mineralisation and bone turnover. Our study group and other researchers have suggested that HIV may impair fracture healing, based on extrapolation from basic science. These observations prompted this study as the true effect of HIV and highly active antiretroviral therapy (HAART) on bone healing is very poorly understood and has not previously been investigated.

Methods: HOST Study is a multicentre case-cohort study being undertaken at two orthopaedic trauma centres in Cape Town, South Africa. All adult patients older than 18 years with fresh (within 2 weeks of injury), closed and open, tibia and femur fractures who undergo intramedullary (IM) nailing for fracture fixation will be eligible for the study. Participants will be recruited over 24 months and undergo a baseline questionnaire, HIV testing and assessment of their bone mineral density (BMD). They will be followed up at 2 and 6 weeks, and at 3, 6, 9 and 12 months. All adult patients who develop delayed bone union at the 6-month follow-up will be considered cases. Adult patients who show evidence of radiological union at 6 months or less will be considered controls. We will then determine if HIV is a risk factor for the development of delayed bone union. HIV prevalence levels in the cases and controls will be summarised using IRR (incidence rate ratio) statistics with their 95\% confidence intervals. Negative binomial regression methods will be used to adjust the IRR estimates for the possible effects of confounding factors and/or important covariates.

Results: Outcomes from the primary manuscript will be disseminated through publications in academic journals and presentations at relevant orthopaedic conferences. We will communicate trial results to all participating sites. Participating sites will communicate results with patients who have indicated an interest in knowing the results.
\end{abstract}

Trial registration number: ClinicalTrials.gov- NCT03131947

Site of study: Groote Schuur Hospital and Tygerberg Hospital, Cape Town, South Africa

Key words: antiretroviral therapy, fracture, femur, human immunodeficiency virus, intramedullary nail, tibia 
Citation: Graham SM, Harrison WJ, Lalloo DG, Simpson AH, Laubscher M, Held M, Ferreira N, Maqungo S. HOST Study - HIV in Orthopaedic Skeletal Trauma Study: protocol for a multicentre case-cohort study SA Orthop J 2018;17(3):53-58. http://dx.doi.org/10.17159/2309-8309/2018/v17n3a7

Editor: Prof LC Marais, University of KwaZulu-Natal, Durban

Received: January $2018 \quad$ Accepted: May $2018 \quad$ Published: August 2018

Copyright: (c) 2018 Graham SM, et al. This is an open-access article distributed under the terms of the Creative Commons Attribution Licence, which permits unrestricted use, distribution and reproduction in any medium, provided the original author and source are credited.

Funding: The funding for this research study was supplied by the Wellcome Trust and AOUK.

Conflict of interest: The authors declare they have no conflicts of interest that are directly or indirectly related to the research.

\section{Introduction}

Worldwide approximately 35.3 million people are HIV positive, with the highest prevalence seen in sub-Saharan Africa. ${ }^{1}$ The introduction of antiretroviral therapy (ART) in 1997 has altered the course and nature of patients infected with HIV by increasing the duration of asymptomatic infection, and consequently patients with HIV are attaining close-to-normal life spans. However, despite these longer life expectancies, there is little evidence to advise the surgeon and patient about the effect of long-term immunosuppression on the fracture repair process in orthopaedic surgery. ${ }^{2}$

HIV principally affects a patient's immunological status by reducing the host CD4 $T$ cell count, resulting in an increase in the risk of a patient developing opportunistic infections. HIV has also been shown to affect other chemical mediators, including interleukins 1 and 6 and tumour necrosis factor (TNF), which have been shown to play a role in the fracture repair process. ${ }^{3-5}$

HIV and ART have both been shown to reduce bone mineral density (BMD), bone mineralisation and bone turnover. ${ }^{6-9}$ In the general population, it has been postulated that a reduced BMD is associated with a reduced speed of fracture healing. ${ }^{10,11}$ If this relationship were to hold true in the context of HIV, then they would not only be at an increased risk of fragility fracture, but also of subsequent delayed fracture healing and failure of fracture fixation.

A major factor known to affect fracture healing is local blood flow to the site of the injury. It is now well established that HIV infection is associated with osteonecrosis, due to interruption in osseous blood supply, although no mechanism for this interruption in blood supply has been shown. ${ }^{12-14}$ ART has also been reported to contribute to this pathology. ${ }^{12}$ Conditions that jeopardise arterial flow to the site of primary bone healing are associated with higher rates of delayed fracture healing and non-union. ${ }^{15-17}$
A small number of studies have investigated the role of HIV in the fracture-healing process. These have suggested that HIV and/ or ART are associated with delayed fracture healing and may result in non-union. ${ }^{11,18}$ The molecular and cellular mechanisms driving this process remain unclear and the true effect of HIV and ART on bone healing is very poorly understood.

Our study group has previously demonstrated an association between HIV infection and impaired fracture healing. 18,19 Furthermore, analysis of patients presenting to Queen Elizabeth Central Hospital, Blantyre, Malawi, for immediate fracture treatment and non-union surgery, indicates twice the frequency of HIV seropositivity in the non-union group (Figure 1) (personal communication - Professor WJ Harrison). Other researchers have suggested that HIV may impair fracture healing, based on extrapolation from basic science. ${ }^{11}$

These observations prompted this study as the true effect of HIV on bone healing is very poorly understood and has not previously been thoroughly investigated.

\section{Study objectives}

Our primary objective is to establish whether HIV is a risk factor for the development of delayed bone union or non-union following a fracture.

Secondary objectives are:

- To assess other risk factors associated with delayed bone union or non-union in HIV-positive and-negative adults, e.g. BMD, ART

- To determine the incidence of superficial, deep and late wound infections in HIV-positive and-negative patients

- To quantify and draw inferences on observed differences in the Disability Rating Index (DRI) and general health-related quality

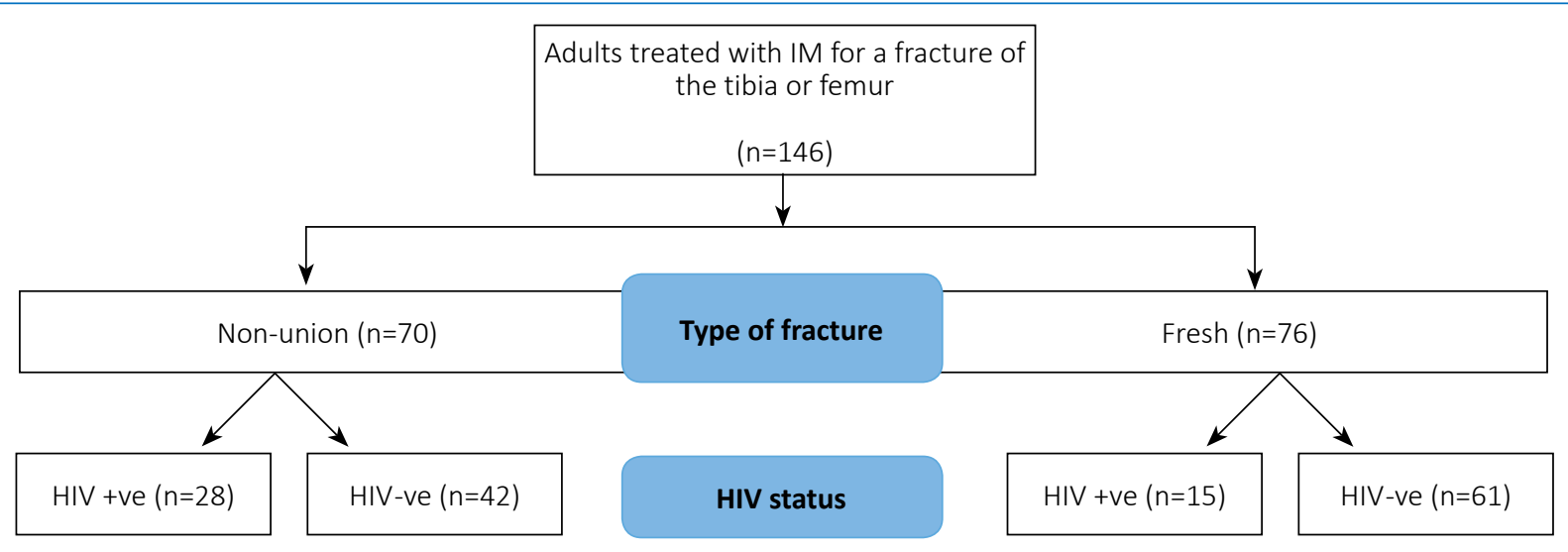

Figure 1. Numbers of patients presenting to QECH for intramedullary nailing for fresh fractures and non-unions: 2005-2007 
of life after fracture healing following IM nailing fracture surgery in HIV-positive and-negative patients.

\section{Methods}

\section{Study design}

HOST Study is a multicentre case cohort study of patients undergoing fracture surgery within the Orthopaedic and Trauma Department at Groote Schuur Hospital (GSH) and Tygerberg Hospital (TBH), Cape Town, South Africa.

\section{Patient selection and recruitment}

Patients older than 18 years of age with fresh (within 2 weeks of injury), closed and open, tibia and femur fractures who undergo IM nailing for fracture fixation will be potentially eligible for inclusion in the study. Participants will undergo a baseline questionnaire, and established risk factors for delayed bone healing and nonunion will be recorded. The patients' full blood count, renal function, vitamin D level and albumin will be assessed. All patients will undergo HIV testing and a measurement of the patients CD4 count and viral load if positive. If a patient is found to have a new diagnosis of HIV, standard local hospital protocol will be followed, and the patient will be offered treatment. If the patient is already known to be HIV positive, their ART regimen will be recorded, if appropriate. Furthermore, all patients will have their BMD measured using a DEXA Heel Scanner, Calscan DXL.

$X$-rays will be performed at follow-up visits or if clinically required. Bone healing will be assessed using a validated $X$-ray scoring system - the Radiological Union Scoring system for the Tibia (RUST scoring system). ${ }^{20,21}$ An independent observer blinded to HIV status will assess radiological fracture union. Participants will be recruited over 24 months.

All adult patients treated with IM nailing of the tibia or femur and who develop delayed bone union at 6 months follow-up will be considered cases. Adult patients who show evidence of radiological union at 6 months or less will be considered controls.

\section{Inclusion criteria}

\section{Patients will be eligible for this study if they:}

- Are older than 18 years of age

- Present within 2 weeks of injury

- Sustained a closed or open fracture of the tibia or femur

- Undergo IM nailing for fracture fixation

\section{Exclusion criteria}

\section{Patients will be excluded from participation in this study in the case of:}

- Major head injury

- Pre-surgical infection at the fracture site

- Open injury for $>48$ hours before the first debridement

- Severe burns

- Pathological fracture

- Evidence that the patient would be unable to adhere to study procedures, complete questionnaires or attend follow-up
Patients who sustain injuries to areas of the body other than the lower limbs, which may affect the primary outcome measure, will have their injuries documented but the participants will still be included in the analysis. For patients with more than one lower limb injury that meets the inclusion criteria, each injury will be included as an individual case and entered separately into the study.

\section{Study outcome}

\section{Primary study outcome:}

- Incidence of delayed bone union in HIV-positive and-negative patients

\section{Secondary study outcomes:}

- Incidence of non-union in HIV-positive and-negative patients

- Other risk factors associated with delayed bone union or nonunion in HIV-positive and-negative adults, e.g. BMD, ART

- Incidence of superficial, deep and late wound infections in HIVpositive and-negative patients

- To quantify and draw inferences on observed differences in the DRI and general health-related quality of life after fracture healing following IM nailing fracture surgery in HIV-positive and -negative patients

\section{Outcome definitions}

\section{Union:}

- Radiological union on RUST score (score of 3 on at least three cortices in AP, lateral, medial or posterior cortex - a total of 9 or more) within 6 months of surgery ${ }^{20,21}$

\section{Delayed bone union:}

- Impaired bone healing at six months (RUST score $<9)^{22-26}$

- Non-union - one or both of the following:

- Need for further surgery to achieve union ${ }^{22,23}$

- Impaired bone healing at nine months (RUST score $<9)^{20,21}$

\section{Superficial wound infection}

- Superficial surgical site infection (SSI)

The Center for Disease Control and Prevention definition of a 'superficial surgical site infection' is a wound infection involving the skin and subcutaneous tissue that occurs after 30 days of surgery (where day $1=$ the procedure date). ${ }^{27}$

- ASEPSIS (28)

ASEPSIS scores $>20$ within 30 days of surgery

\section{Deep wound infection}

The Center for Disease Control and Prevention definition of a 'deep surgical site infection' is a wound infection involving the tissues deep to the skin that occurs within 30 days of injury (closed reduction of fracture) or 90 days (open reduction of fracture). ${ }^{27}$ 


\section{Late wound infection}

This describes any late wound breakdown (>30 days for closed reduction of fractures or $>90$ days for openly reduced fractures) or sinus formation, or unexplained late pain with associated radiological changes consistent with peri-implant sepsis. This will be determined on clinic follow-up for those still under review. Those patients who do not present for follow-up will be phoned and asked about the above.

\section{EuroQol EQ-5D-5L}

The EuroQol EQ-5D is a validated measure of health-related quality of life, consisting of a five-dimension health status classification system and a separate visual analogue scale. ${ }^{23}$ An updated version of the EQ-5D with five response levels, the EQ-5D-5L, has recently been developed to enhance the responsiveness of the instrument to changes in patient health. ${ }^{24}$

\section{Disability Rating Index (DRI)}

The DRI is a self-administered, 12-item Visual Analogue Scale questionnaire assessing the patients' own rating of their disability. ${ }^{22}$ This measure was chosen as it addresses 'gross body movements' rather than specific joints or body segments. Therefore, it will facilitate the assessment of patients with different fractures and injuries of the lower limbs. This outcome measure will not be taken for those patients with longer term (more than 4 weeks) impaired capacity.

\section{Bone mineral density}

- Normal - T score of <1 standard deviations (T score $<-1$ ) below the peak bone mass of a healthy young adult

- Osteopaenia - T score of 1 to 2.5 standard deviations (T score -1 to -2.5 ) below the peak bone mass of a healthy young adult

- Osteoporosis - T score of >2.5 standard deviations ( $T$ score $<-2.5$ ) below the peak bone mass of a healthy young adult

\section{Follow-up}

The full visit-assessment schedule for the study is provided in Table I. Participants will be followed up in the clinic during the 12-month period of follow-up (see study schedule Table I). At 12 months, this may be via phone where appropriate. X-rays will be performed at 3, 6 and 9 months or if clinically indicated.

If a patient is confirmed to have united on RUST score at 3 months, they will be followed up in the clinic at 6 and 9 months and have a telephone consultation at 12 months. The same will be done for patients who are confirmed to have united at 6 and 9 months follow-up.

All patients who have not united by 3,6 or 9 months will have a follow-up clinic visit at 12 months. If there is no evidence of union at 9 months on RUST score, or the patient has undergone a procedure or intervention to assist healing, they will be classified as non-union. The 12-month follow-up will be the final followup time point for the study, either face-to-face or via telephonic communication, even though a patient may need further clinical follow-up for other reasons.

\section{Statistical plan}

\section{Sample size}

Our current register suggests that 400 patients are likely to undergo IM nailing of the tibia and femur at our centres over a two-year study period; $80 \%$ (320) are assumed to be eligible for inclusion.

On the basis of previous research, it is estimated that $85 \%$ (272) of the 320 patients will have union at 6 months (control), and $15 \%$ (48) will have delayed bone union (cases) ${ }^{27,29}$ (Figure 2).

Assuming that $20 \%$ of the controls will be HIV-positive, ${ }^{30}$ the sample size will have $82.8 \%$ power to detect a doubling of HIV prevalence in the cases compared to controls (from 20\% to $40 \%$ at the $5 \%$ significance level).

\section{Statistical methods}

As all consenting patients who present during the study period for IM nailing of the tibia and femur will be recruited, HIV prevalence

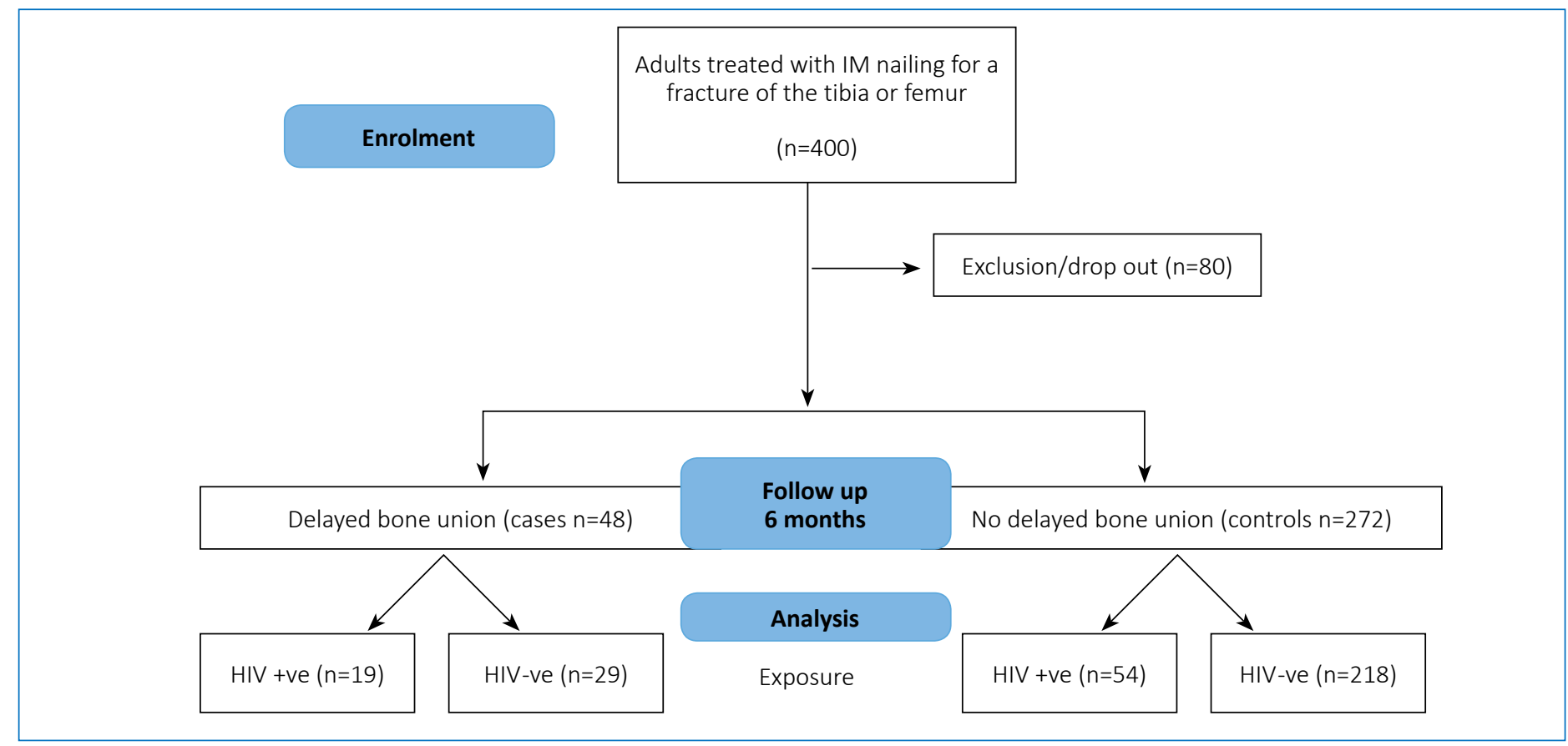


Table I. Summary of data collection plan on electronic database

\begin{tabular}{|c|c|c|c|c|c|c|c|c|}
\hline Classification & $\begin{array}{l}\text { Study measurements/ } \\
\text { Study visits }\end{array}$ & $\begin{array}{l}\text { Base } \\
\text { line }\end{array}$ & 2 weeks & 6 weeks & 3 months & 6 months & 9 months & 12 months \\
\hline Predictor & HIV status/test & $x$ & & & & & & \\
\hline Predictor & $\begin{array}{l}\text { HIV investigations (CD4 count, viral } \\
\text { load), if applicable }\end{array}$ & $x$ & & & $x$ & $x$ & $x$ & \\
\hline Risk factor & $\begin{array}{l}\text { Other lab investigations (FBC, renal } \\
\text { function, albumin, Vit D) }\end{array}$ & $x$ & & & & & & \\
\hline Risk factor & $\begin{array}{l}\text { Socio-demographic characteristics } \\
\& \text { medical history }\end{array}$ & $X$ & & & & & & \\
\hline Risk factor & Body mass index (BMI) & $X$ & & & & & & \\
\hline Risk factor & $\begin{array}{l}\text { Bone mineral density (BMD) } \\
\text { assessment }\end{array}$ & $x$ & & & & & & \\
\hline Intervention & Injury \& treatment detail & $X$ & & & & & & \\
\hline Outcome & Disability Rating Index (DRI) & $x$ & & & $x$ & $x$ & $x$ & \\
\hline Outcome & EQ-5D-5L score & $X$ & & & $x$ & $x$ & $x$ & \\
\hline $\begin{array}{l}\text { Adverse } \\
\text { events }\end{array}$ & ASEPSIS wound score & & $x$ & $x$ & & & & \\
\hline $\begin{array}{l}\text { Adverse } \\
\text { events }\end{array}$ & Infection assessment SSI & & $x$ & $x$ & & & & \\
\hline $\begin{array}{l}\text { Adverse } \\
\text { events }\end{array}$ & Infection assessment DSI & & $x$ & $x$ & $x$ & & & \\
\hline $\begin{array}{l}\text { Adverse } \\
\text { events }\end{array}$ & Infection assessment late infection & & & & & $x$ & $x$ & $x$ \\
\hline $\begin{array}{l}\text { Adverse } \\
\text { events }\end{array}$ & Record complications/interventions & & $x$ & $x$ & $x$ & $x$ & $x$ & $x$ \\
\hline $\begin{array}{l}\text { Concomitant } \\
\text { treatment }\end{array}$ & $\begin{array}{l}\text { Record concomitant ART, NSAID and } \\
\text { steroid use }\end{array}$ & $x$ & $x$ & $x$ & $x$ & $x$ & $x$ & $x$ \\
\hline $\begin{array}{l}\text { Concomitant } \\
\text { treatment }\end{array}$ & Record rehabilitation & & $x$ & $x$ & $x$ & $x$ & $x$ & $x$ \\
\hline \multirow[t]{3}{*}{ Main outcome } & $\begin{array}{l}\text { Radiographic Union Score for Tibial } \\
\text { (RUST) fractures }\end{array}$ & & & $x$ & $x$ & $x$ & $x$ & $x$ \\
\hline & Functional union & & & & $x$ & $x$ & $x$ & $x$ \\
\hline & $\begin{array}{l}\text { Test performed once at baseline or within } 6 \text { weeks } \\
\text { Recorded once only, either at baseline or within } 3 \text { months }\end{array}$ & & & & & & & \\
\hline
\end{tabular}

levels in the cases and controls will be summarised using IRR statistics with their 95\% confidence intervals. Negative binomial regression methods will be used to adjust the IRR estimates for the possible effects of confounding factors and/or important covariates.

\section{Basic descriptive analysis of demographic data and patient characteristics}

Means and standard deviations are used for continuous data along a normal distribution, e.g. age. Level of education will be collected as continuous data and categorised by researchers. It will be analysed using graphs and tables. A confidence interval of $95 \%$ will be used. $P$ values of $<0.05$ are considered statistically significant.

\section{Data management}

Standard operating procedures for data management without breach of confidentiality will be followed. Study participants will be assigned a unique study number at the time of recruitment, under which all electronic data will be captured. From this point, all data will be stored in a password-protected electronic database. Consent forms and case record forms will be kept in a locked filing cabinet in the research office. Only members of the study team (chief investigator, clinical officers and research nurse) will have access to these. Data will be entered into the electronic study database and managed by a data management team. All data exports will be stored on password-protected computers and backed up. The chief investigator and study supervisors will have control of access to the data. Only de-identified data will be exported for analysis.

\section{Conclusion}

This is the largest study of its type to assess the effect of HIV and ART on bone healing. It has been suggested that HIV causes delayed bone union and is a risk factor for the development of non-union following a fracture. If this hypothesis is shown to be true, fracture management could be tailored to optimise bone union during the fracture-healing phase in HIV-positive patients, improving outcomes and reducing the substantial social burden that occurs in these patients. ${ }^{31}$ If this hypothesis is shown to be incorrect then this study will demonstrate that HIV-positive patients can be treated in the same way as HIV-negative patients when managing orthopaedic skeletal trauma. 


\section{Ethics statement}

The HOST study has received local and the University of Cape Town and University of Stellenbosch Faculty of Health Science Human Ethics Committee approval - reference number 590/2016 and N17/05/052.

The author/s declare that this submission is in accordance with the principles laid down by the Responsible Research Publication Position Statements as developed at the 2 nd World Conference on Research Integrity in Singapore, 2010.

No other intervention will be performed other than the standard treatment according to our unit's policies and current best evidence. HIV testing is offered to all patients of our study units, based on the recommendations of the Department of Health HIV counselling and Testing Policy Guidelines.

All study team members will undergo good clinical practice training prior to commencement of study. Informed consent will be obtained from all study participants, either written or witnessed verbal consent, with thumbprint if the participant is illiterate. Since a substantial proportion of participants will be HIV positive, every effort will be made to safeguard patient confidentiality as outlined above.

\section{References}

1. Joint United Nations Programme on HIV/AIDS (UNAIDS). Global report: UNAIDS report on the global AIDS epidemic 2013. 2013.

2. Chen LF, Hoy J, Lewin SR. Ten years of highly active antiretroviral therapy for HIV infection. Med J Aust. 2007 Feb 5;186(3):146-51.

3. Hankemeier S, Grässel S, Plenz G, Spiegel HU, Bruckner P, Probst A. Alteration of fracture stability influences chondrogenesis, osteogenesis and immigration of macrophages. J Orthop Res. $2001 \mathrm{Jul} ; \mathbf{1 9}(4): 531-38$.

4. Hauser $C J$, Zhou $X$, Joshi P, Cuchens MA, Kregor P, Devidas M, et al. The immune microenvironment of human fracture/soft-tissue hematomas and its relationship to systemic immunity. J Trauma. 1997 May;42(5):895-903-904.

5. Bongiovanni $M$, Tincati $C$. Bone diseases associated with human immunodeficiency virus infection: pathogenesis, risk factors and clinical management. Curr Mol Med. 2006 Jun;6(4):395-400.

6. Singh K, Moyle GJ. Bone mineral abnormalities in persons with HIV infection: signal or noise? AIDS Read. 2006 Aug;16(8):40710, 413-18.

7. Soyka LA, Fairfield WP, Klibanski A. Clinical review 117: Hormonal determinants and disorders of peak bone mass in children. J Clin Endocrinol Metab. 2000 Nov;85(11):3951-63.

8. Mondy K, Tebas P. Emerging bone problems in patients infected with human immunodeficiency virus. Clin Infect Dis. $2003 \mathrm{Apr}$ 1;36(Suppl 2):S101-105.

9. Mondy K, Yarasheski K, Powderly WG, Whyte M, Claxton S, DeMarco D, et al. Longitudinal evolution of bone mineral density and bone markers in human immunodeficiency virus-infected individuals. Clin Infect Dis. 2003 Feb 15;36(4):482-90.

10. Giannoudis P, Tzioupis C, Almalki T, Buckley R. Fracture healing in osteoporotic fractures: is it really different? A basic science perspective. Injury. 2007 Mar;38 Suppl 1:S90-99.

11. Chokotho L, Harrison WJ, Lubega N, Mkandawire NC. Avascular necrosis of the femoral head in HIV positive patients-an assessment of risk factors and early response to surgical treatment. Malawi Med J. 2013 Jun;25(2):28-32.

12. Matos MA, Alencar RW de, Matos SS da R. Avascular necrosis of the femoral head in HIV infected patients. Braz J Infect Dis. 2007 Feb;11(1):31-4.

13. Monier P, McKown K, Bronze MS. Osteonecrosis complicating highly active antiretroviral therapy in patients infected with human immunodeficiency virus. Clin Infect Dis. 2000 Dec;31(6):1488-92.

14. Wallace AL, Draper ER, Strachan RK, McCarthy ID, Hughes SP. The effect of devascularisation upon early bone healing in dynamic external fixation. J Bone Joint Surg Br. 1991 Sep;73(5):819-25.
15. Dickson KF, Katzman S, Paiement G. The importance of the blood supply in the healing of tibial fractures. Contemp Orthop. 1995 Jun;30(6):489-93.

16. Hausman MR, Schaffler MB, Majeska RJ. Prevention of fracture healing in rats by an inhibitor of angiogenesis. Bone. 2001 Dec;29(6):560-64.

17. Harrison WJ, Lewis CP, Lavy CBD. Wound healing after implant surgery in HIV-positive patients. J Bone Joint Surg Br. 2002 Aug;84(6):802-806.

18. Richardson J, Hill AM, Johnston CJC, McGregor A, Norrish AR, Eastwood D, et al. Fracture healing in HIV-positive populations. J Bone Joint Surg Br. 2008 Aug;90(8):988-94.

19. Harrison WJ, Lewis CP, Lavy CBD. Open fractures of the tibia in HIV positive patients: a prospective controlled single-blind study. Injury. 2004 Sep;35(9):852-56.

20. Kooistra BW, Dijkman BG, Busse JW, Sprague S, Schemitsch EH, Bhandari $M$. The radiographic union scale in tibial fractures: reliability and validity. J Orthop Trauma. 2010 Mar;24 Suppl 1:S81-86.

21. Whelan DB, Bhandari M, Stephen D, Kreder H, McKee MD, Zdero $\mathrm{R}$, et al. Development of the radiographic union score for tibial fractures for the assessment of tibial fracture healing after intramedullary fixation. J Trauma. 2010 Mar;68(3):629-32.

22. Winquist RA, Hansen ST, Clawson DK. Closed intramedullary nailing of femoral fractures. A report of five hundred and twenty cases. J Bone Joint Surg Am. 1984 Apr;66(4):529-39.

23. Court-Brown CM, Caesar B. Epidemiology of adult fractures: $A$ review. Injury. 2006 Aug;37(8):691-97.

24. Keating JF, O’Brien PJ, Blachut PA, Meek RN, Broekhuyse HM. Locking intramedullary nailing with and without reaming for open fractures of the tibial shaft. A prospective, randomized study. J Bone Joint Surg Am. 1997 Mar;79(3):334-41.

25. Gustilo RB, Mendoza RM, Williams DN. Problems in the management of type III (severe) open fractures: a new classification of type III open fractures. J Trauma. 1984 Aug;24(8):742-46.

26. Study to Prospectively Evaluate Reamed Intramedullary Nails in Patients with Tibial Fractures Investigators, Bhandari M, Guyatt G, Tornetta P, Schemitsch EH, Swiontkowski M, et al. Randomized trial of reamed and unreamed intramedullary nailing of tibial shaft fractures. J Bone Joint Surg Am. 2008 Dec;90(12):2567-78.

27. Horan TC, Andrus M, Dudeck MA. CDC/NHSN surveillance definition of health care-associated infection and criteria for specific types of infections in the acute care setting. Am J Infect Control. 2008 Jun;36(5):309-32.

28. Wilson AP, Treasure T, Sturridge MF, Grüneberg RN. A scoring method (ASEPSIS) for postoperative wound infections for use in clinical trials of antibiotic prophylaxis. Lancet. 1986 Feb 8;1(8476):311-13.

29. Drosos GI, Bishay M, Karnezis IA, Alegakis AK. Factors affecting fracture healing after intramedullary nailing of the tibial diaphysis for closed and grade I open fractures. J Bone Joint Surg Br. 2006 Feb;88(2):227-31.

30. Bates J, Mkandawire N, Harrison WJ. The incidence and consequences of early wound infection after internal fixation for trauma in HIV-positive patients. J Bone Joint Surg Br. 2012 Sep;94(9):1265-70.

31. Harrison WJ. HIV/AIDS in trauma and orthopaedic surgery. J Bone Joint Surg Br. 2005 Sep;87(9):1178-81. 